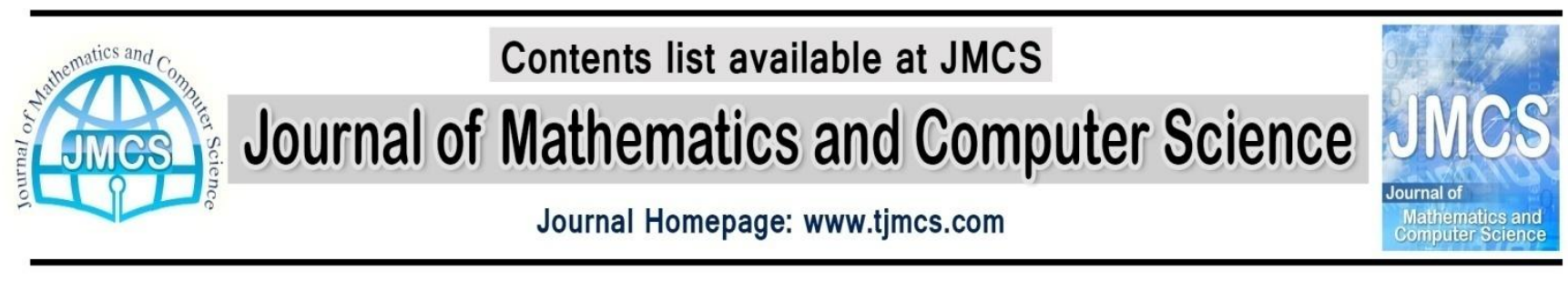

\title{
Model Reduction by Hermite Polynomials and Genetic Algorithm
}

\author{
Hasan Nasiri Soloklo \\ Department of Electrical \\ Engineering, Firoozkooh \\ Branch, Islamic Azad \\ University, Firoozkooh, Iran \\ hasannasirisoloklo@yahoo.c \\ om \\ Article history: \\ Received August 2013 \\ Accepted November 2013 \\ Available online November 2013
}

\author{
Omid Nali \\ Department of Electrical \\ Engineering, Saghez branch, \\ Islamic Azad university, \\ Saghez, Iran \\ omid.nali@gmail.com
}

\author{
Malihe. M. Farsangi \\ Electrical Engineering \\ Department, Shahid Bahonar \\ University of Kerman
}

mmaghfoori@mail.uk.ac.ir

\begin{abstract}
The present paper attempts to develop order reduction methods where the suggested reduction model consists of two phases. First, full order system is expanded by Hermite polynomials, then a set of parameters in a fixed structure are determined, whose values define the reduced order system. The values are obtained using Genetic Algorithm (GA) by minimizing the errors between the $l$ first coefficients of Hermite polynomials expansion of full and reduced systems. To satisfy the stability, Routh criterion is used as constraints in optimization problem. To present the ability of the proposed method, three test systems are reduced. The results obtained are compared with other existing techniques. The results obtained show the accuracy and efficiency of the proposed method.
\end{abstract}

Keywords: Hermite polynomials, genetic algorithm, Routh array, order reduction, stability constraints.

\section{Introduction}

Various methods are reported in the literature for order reduction in time domain and frequency domain. Model reduction started by Davison in 1966 [1] and followed by Chidambara by suggestion several modifications to Davison's approach [2]-[4]. After that different approaches proposed using dominant pole retention or dominant eigenvalue retention [5], Routh approximation [6], Hurwitz polynomial approximation [7], [8], stability equation method [9], [10], moments matching [11]-[14], continued fraction method [15]-[17] and Pade approximation [18] and etc.

The issue of optimality in model reduction was considered by Wilson [19], [20] who suggested an optimization approach based on minimization of the integral squared impulse response error between full and reduced-order models. This attempt was continued by other researches through other approaches [21]-[24]. 
In 1981 [25], the controllability and observability of the states was considered in model reduction by Moore. The suggested approach suffered from steady state errors but the stability of the reduced model was assured if the original system was also stable [26]. Furthermore, the concept of $H_{\infty}, H_{2}, L_{2}$ and $L_{\infty}$ were used for model reduction in [27]-[30].

In recent decade, the evolutionary techniques such as Particle Swarm Optimization (PSO) and Genetic Algorithm (GA) are used for order reduction of systems [31]-[33]. In these approaches, the reduced order model's parameters are achieved by minimizing a fitness function which is often Integral Square Error (ISE), Integral Absolute Error (IAE), $H_{2}$ norm or $H_{\infty}$ norm [34]-[36].

This paper introduces a new alternative method for order reduction using orthogonal polynomials through Hermite polynomials. In this method, the full order system is expanded by Hermite polynomials and then the $l$ first coefficients of Hermite polynomials are obtained. A desire fixed structure for reduced order model is considered and a set of parameters are defined, whose values determine the reduced order system. These unknown parameters are determined using GA by minimizing the errors between the $l$ first coefficients of Hermite polynomials expansion of full and reduced systems. To satisfy the stability, Routh criterion is applied as it is used in [37] where, it states in optimization problem as constraints and subsequently, optimization problem converted to a constrained optimization problem. To show the accuracy of the proposed method, three systems are reduced by the proposed method and compared with those available in the literature.

To make a proper background, Hermite polynomials and GA are briefly explained in Sections 2 and 3, respectively. The proposed method is explained in Section 4. The ability of the proposed approach is shown in Section 5. The paper is concluded in Section 6. Finally, the references are introduced.

\section{The Hermite polynomials}

The Hermite polynomials are a class of orthogonal polynomials [38]. A Hermite polynomial $H_{n}$ in $x$ of degree on $n$ is defined as:

$H_{n}(x)=(-1)^{n} e^{x^{2}} \frac{d}{d x^{n}}\left(e^{-x^{2}}\right)$

where $\frac{d}{d x^{n}}$ represents differential operator.

Also, the Hermite polynomials can be obtained by recursive formula as follows:

$$
H_{n+1}(x)=2 x H_{n}(x)-2 n H_{n-1}(x)
$$

in which $H_{0}(x)=1$ and $H_{1}(x)=2 x$.

The Hermite polynomials are orthogonal on the interval $(-\infty,+\infty)$ with respect to the weight function $W(x)$, where $W(x)$ is expressed as

$W(x)=e^{-x^{2}}$

By the above definitions, we have the following:

$$
\int_{-\infty}^{+\infty} W(x) H_{m}(x) H_{n}(x) d x=\left\{\begin{array}{cc}
2^{n} n ! \sqrt{\pi} \quad m=n \\
0 \quad m \neq n
\end{array}\right.
$$

Therefore, a piecewise continuous function, $f(x)$, can be expanded as:

$$
\sum_{n=0}^{\infty} C_{n} H_{n}(x)=\left\{\begin{array}{c}
f(x) \quad f(x) \text { is continous } \\
\frac{f\left(x^{-}\right)+f\left(x^{+}\right)}{2} \quad \text { at discountinuos poin } \mathrm{t}
\end{array}\right.
$$


where $C_{n}$ are coefficients of Hermite polynomial that are obtained by following equation:

$C_{n}=\frac{1}{2^{n} n ! \sqrt{\pi}} \int_{-\infty}^{+\infty} e^{-x^{2}} f(x) H_{n}(x) d x$

Thus, by considering the first $l$ terms of (5), a good approximant of $f(x)$ is obtained.

\section{Genetic algorithm}

GA is a search algorithm based on the mechanism of genetic and natural selection. The GAs start with random generation of initial population and then the selection, crossover and mutation are preceded until the maximal generation is reached. A typical simple genetic algorithm is described in detail in [39]. The principle of GA is shown by Fig. 1.

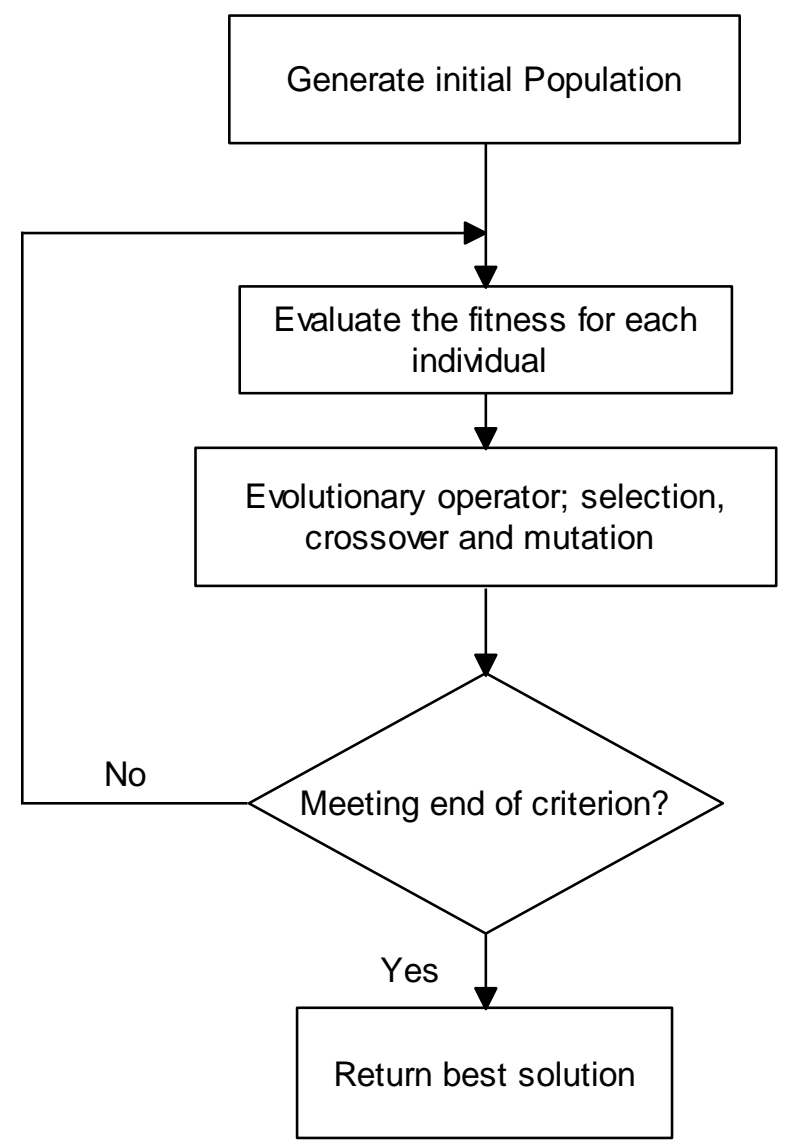

Figure 1: General principle of GA.

\section{The Proposed model reduction method}

Let

$G(s)=\frac{a_{1} s^{n-1}+a_{2} s^{n-2}+\ldots+a_{n}}{s^{n}+b_{1} s^{n-1}+b_{2} s^{n-2}+\ldots+b_{n}}$ 
be an $n$th order single-input single-output (SISO) system which is strictly proper and asymptotically stable where $a_{i}$ and $b_{i}$ are constants. It is desired a stable $r$ th order approximant (that $r$ is smaller than n) to (7) as

$G_{r}(s)=\frac{c_{1} s^{r-1}+c_{2} s^{r-2}+\ldots+c_{r}}{s^{r}+d_{1} s^{r-1}+d_{2} s^{r-2}+\ldots+d_{r}}$

such that the principal and important specifications of the full order system are retained in the reduced order model. In (8) $c_{i}$ and $d_{i}$ are unknown constants.

To obtain the reduced model by the proposed method, firstly, the full order system is expanded based on Hermite polynomials expansion. Then the $l$ first coefficients of Hermite polynomials expansion of original system are obtained and shown by $C_{i}, i=0,1, \ldots, l$. Then a desire fixed structure is considered for reduced order model as defined in (8) where $c_{i}$ and $d_{i}$ are unknown parameters of reduced order model that are obtained by GA. The goal of the optimization is to find the best parameters for $G_{r}(s)$. Therefore, each chromosome is a $d$-dimensional vector in which $d$ is $c_{i}+d_{i}$. Each chromosome is a solution to $G_{r}$ and for each solution (chromosome), the Hermite polynomials are obtained. Each chromosome in the population is evaluated by minimizing the following fitness function:

$J^{*}=\sum_{i=0}^{l}\left(\left|C_{i}-\hat{C}_{i}\right|\right)$

in which, $\hat{C}_{i}$ are the coefficients of Hermite polynomials of reduced order system. The algorithm searches for the best chromosome until the termination criteria are met. At this stage the best parameters are given as parameters of reduced order model.

Furthermore, the reduced model must be stable if the original system is stable. Therefore, the Routh criterion is applied to assure the stability. For specifying the stability conditions, first, the denominator of reduced order model in (8) is shown as below [40]:

$$
\begin{aligned}
& s^{r}+h_{1} s^{r-1}+\left(h_{2}+h_{3}+\ldots+h_{r}\right) s^{r-2}+h_{1}\left(h_{3}+h_{4}+\ldots+h_{r}\right) s^{r-3}+ \\
& {\left[h_{2}\left(h_{4}+h_{5}+\ldots+h_{r}\right)+h_{3}\left(h_{5}+h_{6}+\ldots+h_{r}\right)+\right.} \\
& h_{4}\left(h_{6}+h_{7}+\ldots+h_{r}\right)+\ldots
\end{aligned}
$$

which is constructed by taking the coefficients of the first two rows of the Routh array with the elements of its first column given by

$$
1, h_{1}, h_{2}, h_{1} h_{3}, h_{2} h_{4}, h_{1} h_{3} h_{5}, \ldots, h_{1+k} h_{3+k} \ldots h_{r-2} h_{r}
$$

where $k$ is equal to 1 for even $r$ and $k$ is equal to 0 for odd $r$.

Comparing the entries of the first row with $1, d_{2}, d_{4}, \ldots$ and those of the second row with $d_{1}, d_{3}, d_{5}, \ldots$, the following relations are obtained:

$$
\begin{aligned}
& d_{1}=h_{1} \\
& d_{2}=\left(h_{2}+h_{3}+\ldots+h_{r}\right) \\
& d_{3}=h_{1}\left(h_{3}+h_{4}+\ldots+h_{r}\right) \\
& \vdots \\
& d_{r}=\left(h_{1+k} h_{3+k} h_{r-2} h_{r}\right)
\end{aligned}
$$

Substituting the above relations in reduced order model's denominator, (10) is achieved. Therefore, the necessary and sufficient condition for all the poles of the reduced system to be strictly in the left-half plane is 


$$
\begin{aligned}
& h_{1}>0 \\
& h_{2}>0 \\
& \vdots \\
& h_{r}>0
\end{aligned}
$$

and subsequently

$$
\begin{aligned}
& d_{1}>0 \\
& d_{2}>0 \\
& \vdots \\
& d_{r}>0
\end{aligned}
$$

Thus, to have a stable reduced system, the reduced order model's parameters are determined by minimizing the following fitness function:

$$
\begin{aligned}
& J=\sum_{i=0}^{l}\left(\left|C_{i}-\hat{C}_{i}\right|\right) \\
& \text { subject to } d_{j}>0 \quad \text { for } j=1, \cdots, r
\end{aligned}
$$

\section{Simulations and results}

To assess the efficiency of the proposed approach, it has been applied on three test systems, where a step-by-step procedure is given for the first test system.

Test system 1: The first system to be reduced is a system of order 6, given by Mukherjee where a procedure was presented in [41] to obtain reduced system using response- matching technique. The system is as follows:

$$
G(s)=\frac{s^{5}+1014 s^{4}+14069 s^{3}+69140 s^{2}+140100 s+100000}{s^{6}+222 s^{5}+14541 s^{4}+248420 s^{3}+1454100 s^{2}+2220000 s+1000000}
$$

The reduced order model can be achieved by the following steps, using Hermite polynomials and GA: Step 1: Based on section 2, the Hermite polynomials of the full order system in (16) are obtained as:

$$
\begin{aligned}
G(s)= & (0.0387) \times 1+(0.0197) \times(2 s)+(-0.0014) \times\left(4 s^{2}-2\right)+(-0.0019) \times\left(8 s^{3}-12 s\right)+ \\
& \left(1.1702 \times 10^{-4}\right) \times\left(16 s^{4}-48 s^{2}+12\right)+\left(1.5412 \times 10^{-4}\right) \times\left(32 s^{5}-160 s^{3}+120 s\right)+\cdots
\end{aligned}
$$

Step 2: The full order of the system represented in (16) is going to be reduced to a third-order system with the following transfer function:

$$
G_{r}(s)=\frac{c_{0} s^{2}+c_{1} s+c_{2}}{s^{3}+d_{0} s^{2}+d_{1} s+d_{2}}
$$

where $c_{i}$ and $d_{i}$ are unknown parameters of reduced order model.

Step 3: GA is applied to obtain the unknown parameters in (18). Since, the goal of the optimization is to find the best parameters for $G_{r}(s)$, therefore, a configuration is considered with six genes $\left(c_{i}+d_{i}\right)$. The number of chromosomes for a population is set to be 100 .

The chromosomes evolve through successive iterations, called generations. During each generation, the chromosomes are evaluated with some measure of fitness, which is calculated from the objective function (15).

Moving to a new generation is done from the results obtained for the old generation. A based roulette wheel is created from the obtained values of the objective function of the current population. To create the next generation, new chromosomes, called off spring, are formed using a crossover operator and a mutation operator. In this paper, one point crossover is applied with the crossover probability $p_{c}=0.9$ 
and the mutation probability is selected to be changed linearly from $p_{m}=0.05$ to $p_{m}=0.005$. Also, the number of iteration is considered to be 100 .

Each chromosome is a solution to $G_{r}$ and for each solution (chromosome), the Hermite polynomials are obtained. Each chromosome in the population is evaluated using the objective function defined by (15) searching for the best $J$ until the termination criteria is met. At this stage the best parameters are given for reduced order model where, the following reduced order model is obtained:

$$
G_{\text {Hermite }}=\frac{4.56 s^{2}+26.96 s+50.74}{s^{3}+53.49 s^{2}+690.6 s+507.02}
$$

The Hermite polynomials of obtained reduced order model are as:

$$
\begin{gathered}
G_{\text {Hermite }}(s)=(0.0387) \times 1+(0.0197) \times(2 s)+(-0.0014) \times\left(4 s^{2}-2\right)+(-0.0019) \times\left(8 s^{3}-12 s\right)+ \\
\left(1.693 \times 10^{-4}\right) \times\left(16 s^{4}-48 s^{2}+12\right)+\left(1.5410 \times 10^{-4}\right) \times\left(32 s^{5}-160 s^{3}+120 s\right)+\cdots
\end{gathered}
$$

Comparing (17) and (20) shows that the best approximant of $G(s)$ is achieved. The step response of the full order system and that of the system with third-order reduced models are shown in Fig. 2. This figure shows that the obtained reduced order model is an adequate low-order model that retains the characteristics of full order model. Also, to show the efficiency of the proposed method, the step and frequency responses of the obtained reduced model are compared with those available in the literature. Figs. 3 - 4, show the comparison of the results obtained with the proposed method by Mukherjee [41], Optimal Hankel norm approximation (HSV) [42] and Balanced Truncation (BT) [42], respectively.

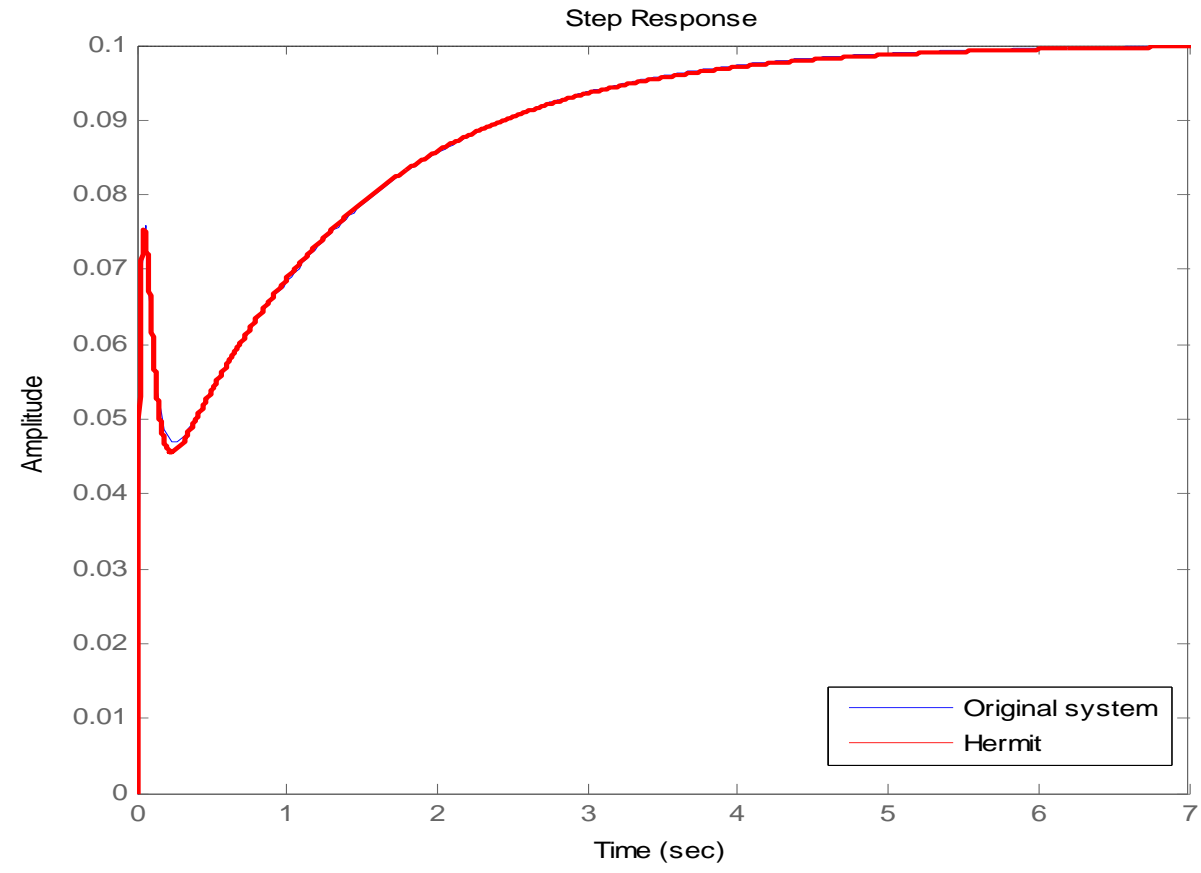

Figure 2: The step response of full order and reduced order model by the proposed method for test system 1. 


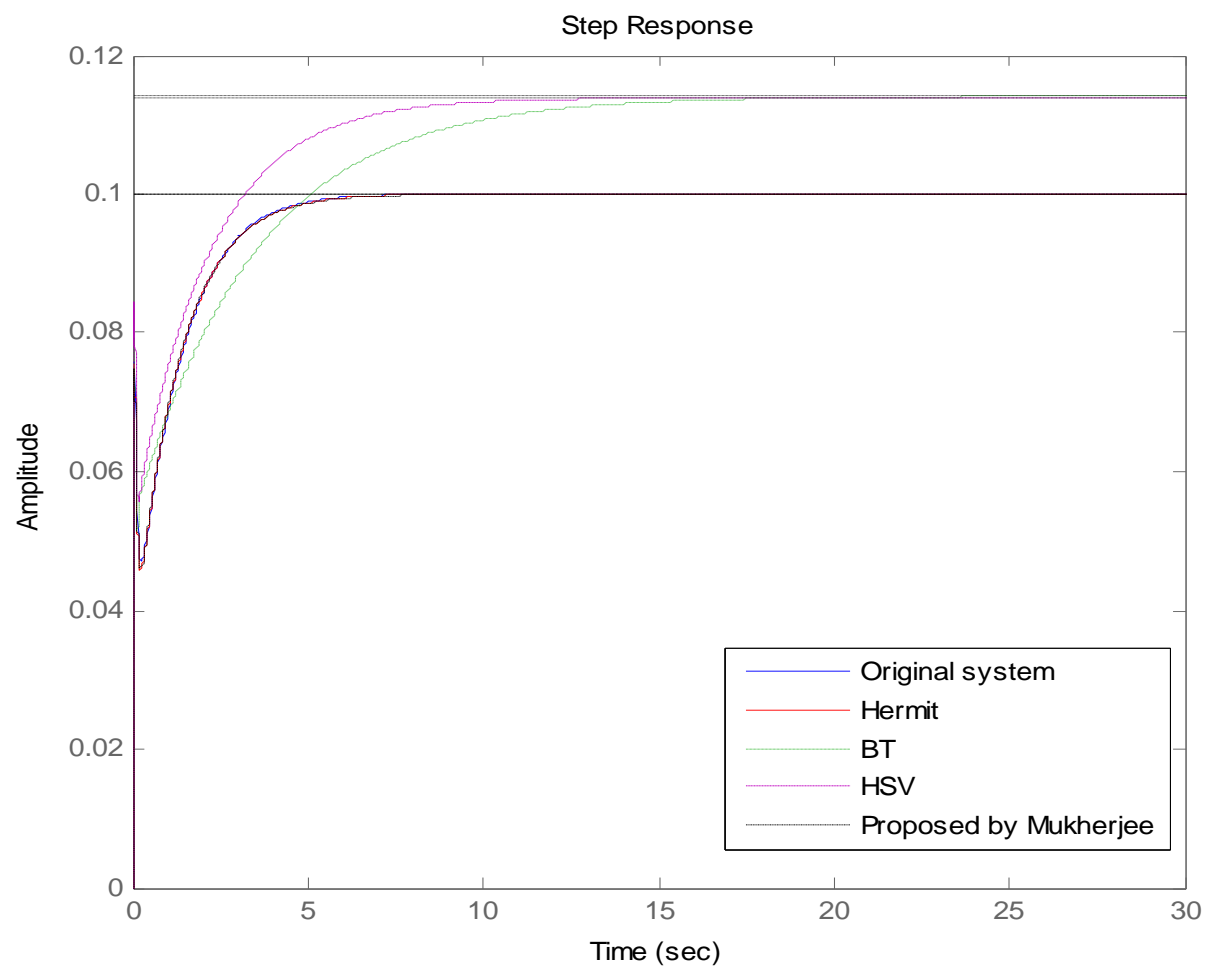

Figure 3: The step response of full order and reduced order model by the proposed method and other methods for test system 1.

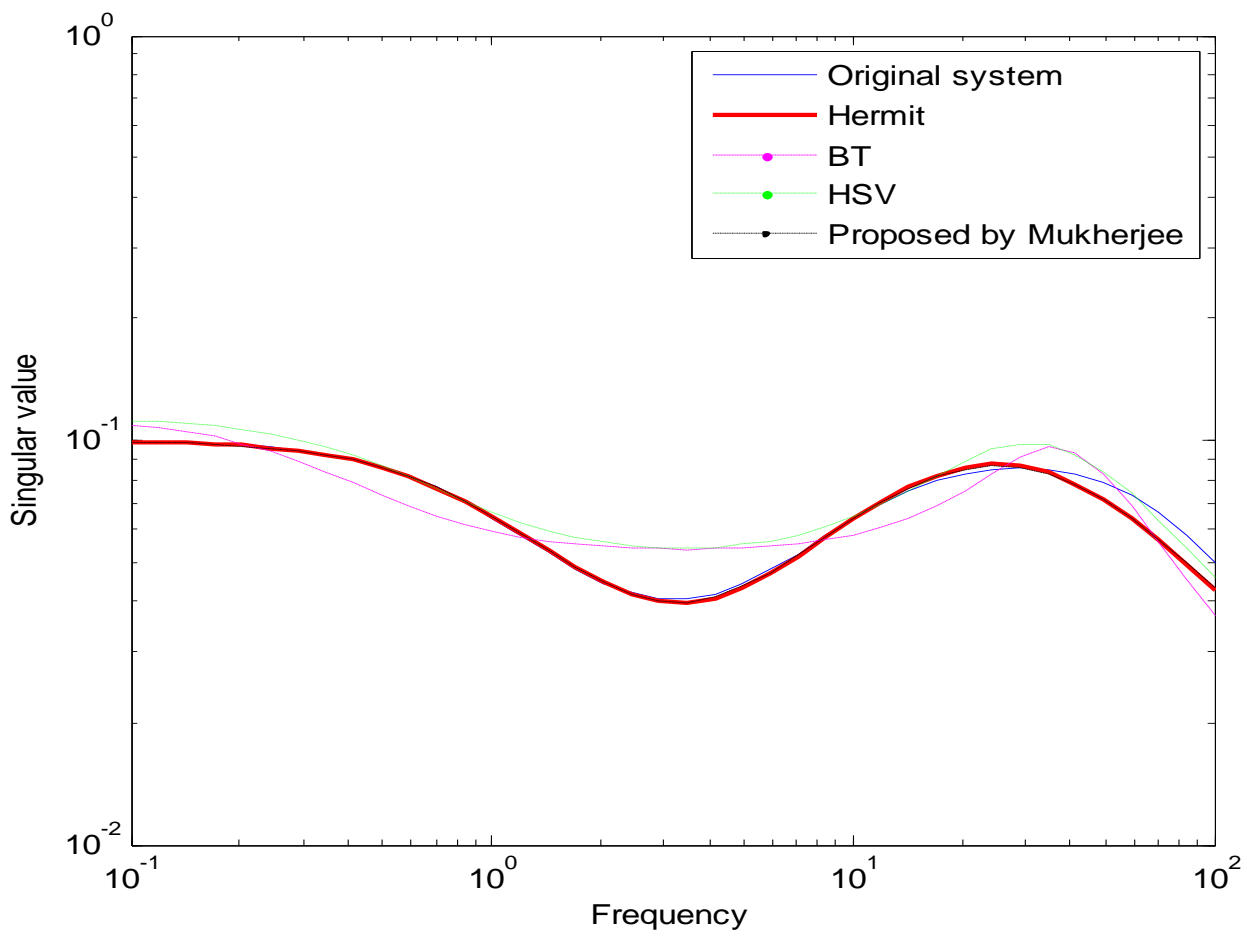

Figure 4: The frequency response of full order and reduced order model by the proposed method and other methods for test system 1. 
These figures show that the achieved results from the proposed method and the suggested method by Mukherjee are very similar to original system comparing to HSV and BT methods. The steady-state gains of full and reduced systems should be equal since it is a very important requirement for model reduction. Also, the frequency responses of full and reduced systems are the same, which will make the stability and performance characteristics of both systems to be the same.

Furthermore, the specifications of the proposed method such as steady state value and maximum overshoot are obtained and compared with the suggested method by Mukherjee [41], HSV and BT. The results are shown in Table 1 . Also, $H_{\infty}$ norm of the error between the step responses of full order and reduced order models $\left(e=\left|y-y_{r}\right|\right)$ is given in Table 1 . It is clearly seen that the specifications of reduced order model that is achieved by the proposed method and the one by Mukherjee are close to the specifications of original system.

Table1. Comparison of methods for test system 1.

\begin{tabular}{|c|c|c|c|c|}
\hline Model & Steady state value & Overshoot (\%) & ISE & $\begin{array}{c}\text { Infinity norm of } \\
\text { error }\end{array}$ \\
\hline Original system & 0.1 & 0 & - & - \\
\hline Hermite - GA & 0.1 & 0 & $2.5 \times 10^{-6}$ & 0.0069 \\
\hline $\begin{array}{c}\text { Proposed by } \\
\text { Mukherjee }\end{array}$ & 0.1 & 0 & $1.34 \times 10^{-6}$ & 0.0074 \\
\hline BT & 0.114 & 0 & 0.0379 & 0.0141 \\
\hline HSV & 0.114 & 0 & 0.0375 & 0.0139 \\
\hline
\end{tabular}

Also, the plot of $e=\left|y-y_{r}\right|$ is given for the full order and reduced systems in Fig. 5. This figure illustrates that the obtained error by the proposed method and the one by Mukherjee is very similar and less than HSV and BT methods.

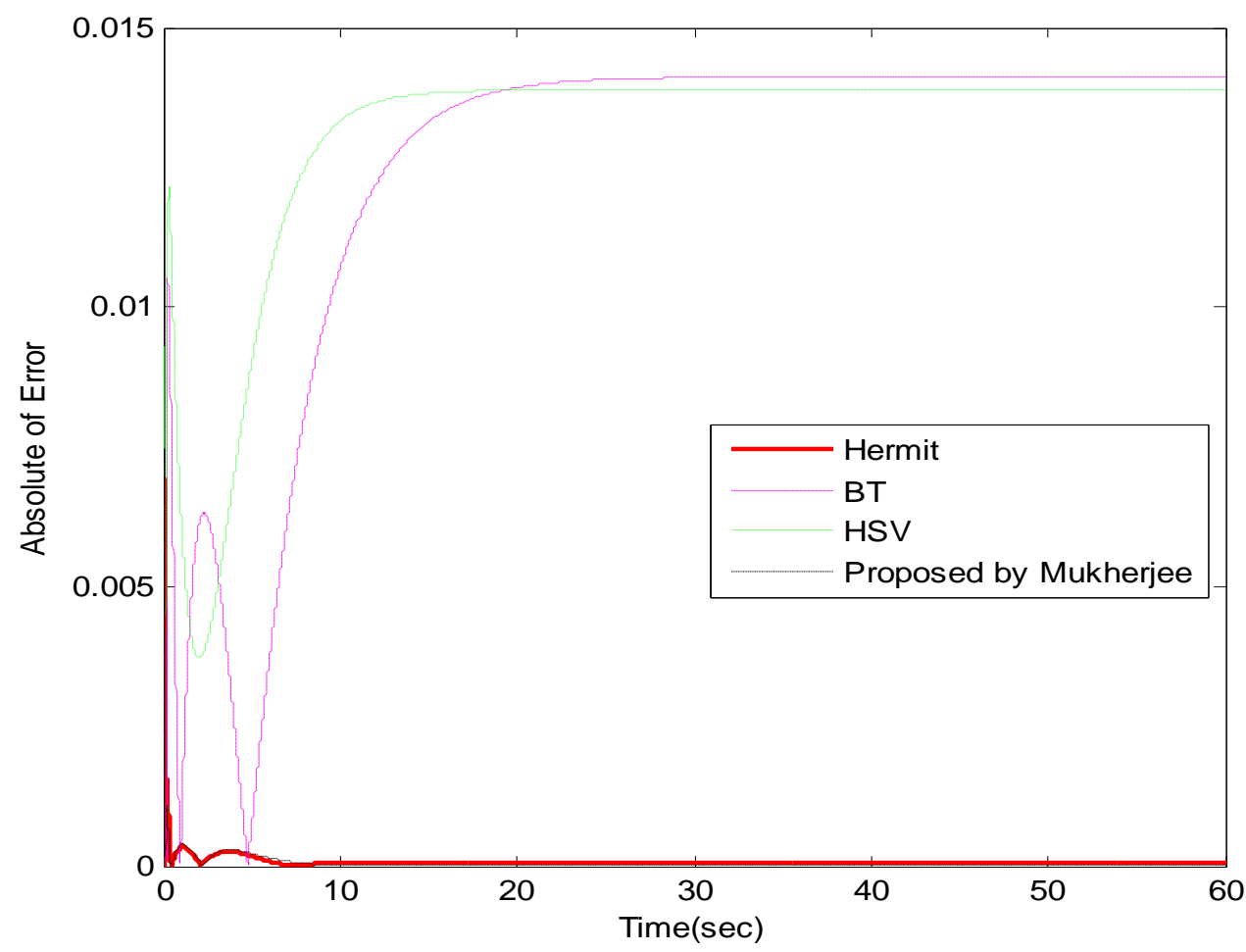

Figure 5: The plot of $e=\left|y-y_{r}\right|$ for the full order and reduced systems by the proposed method and other methods for test system 1. 
Test system 2: In [40], a procedure is presented to obtain a reduced order system by Routh-Pade approximation using Luus-Jaakola algorithm. To compare the proposed method with Luus-Jaakola algorithm, the system given in [40] is adopted which is a third-order system:

$$
G=\frac{8 s^{2}+6 s+2}{s^{3}+4 s^{2}+5 s+2}
$$

Based on the explanations given for test system 1, the obtained reduced system by the proposed method is as follows:

$$
G_{\text {Hermite }}=\frac{5.97 s+4.172}{s^{2}+2.58 s+4.176}
$$

The step and frequency responses of the original system and the obtained reduced model are shown in Figs. 6-7. In these figures, the responses of the system with second-order primary reduced models obtained by other methods are also included for comparison. Also, the plot of $e=\left|y-y_{r}\right|$ is given for the full order and reduced systems in Fig. 8.

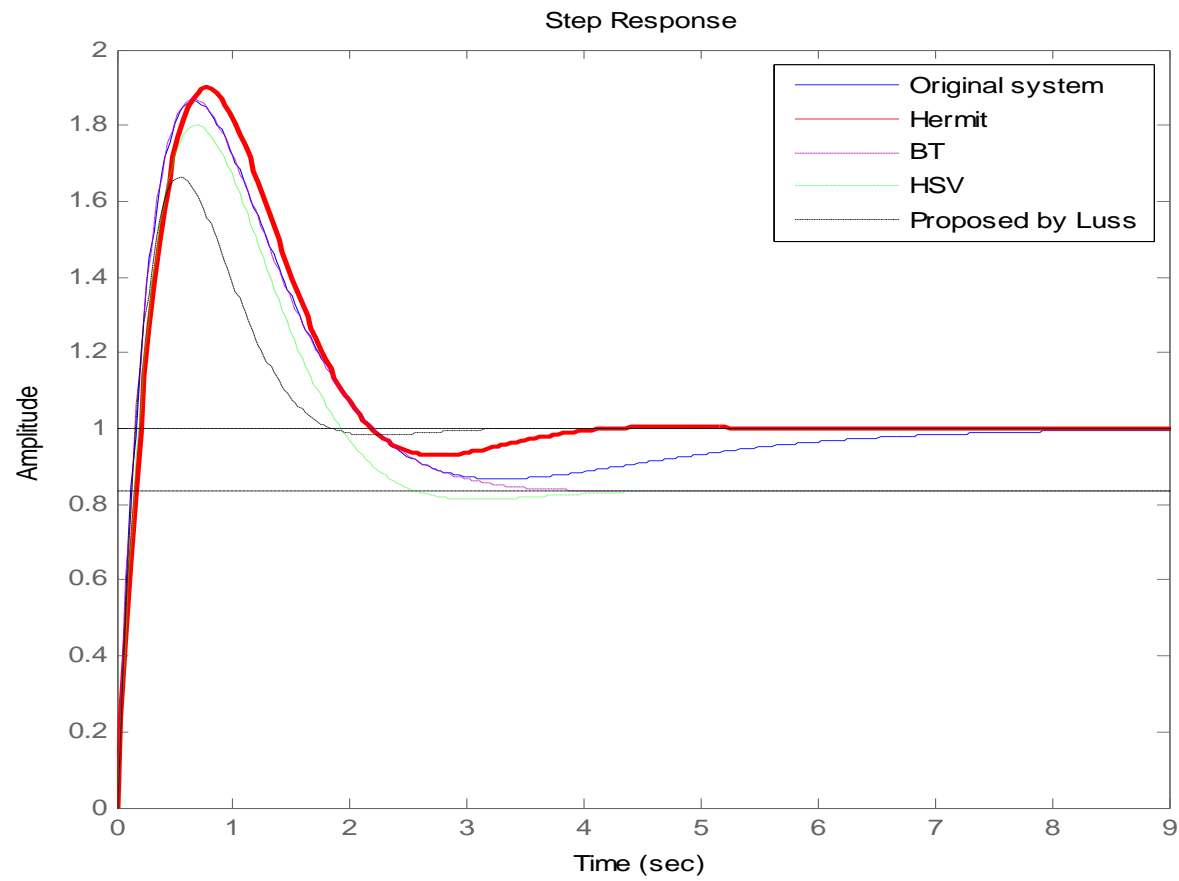

Figure 6: The step response of full order and reduced order model by the proposed method and other methods for test system 2. 


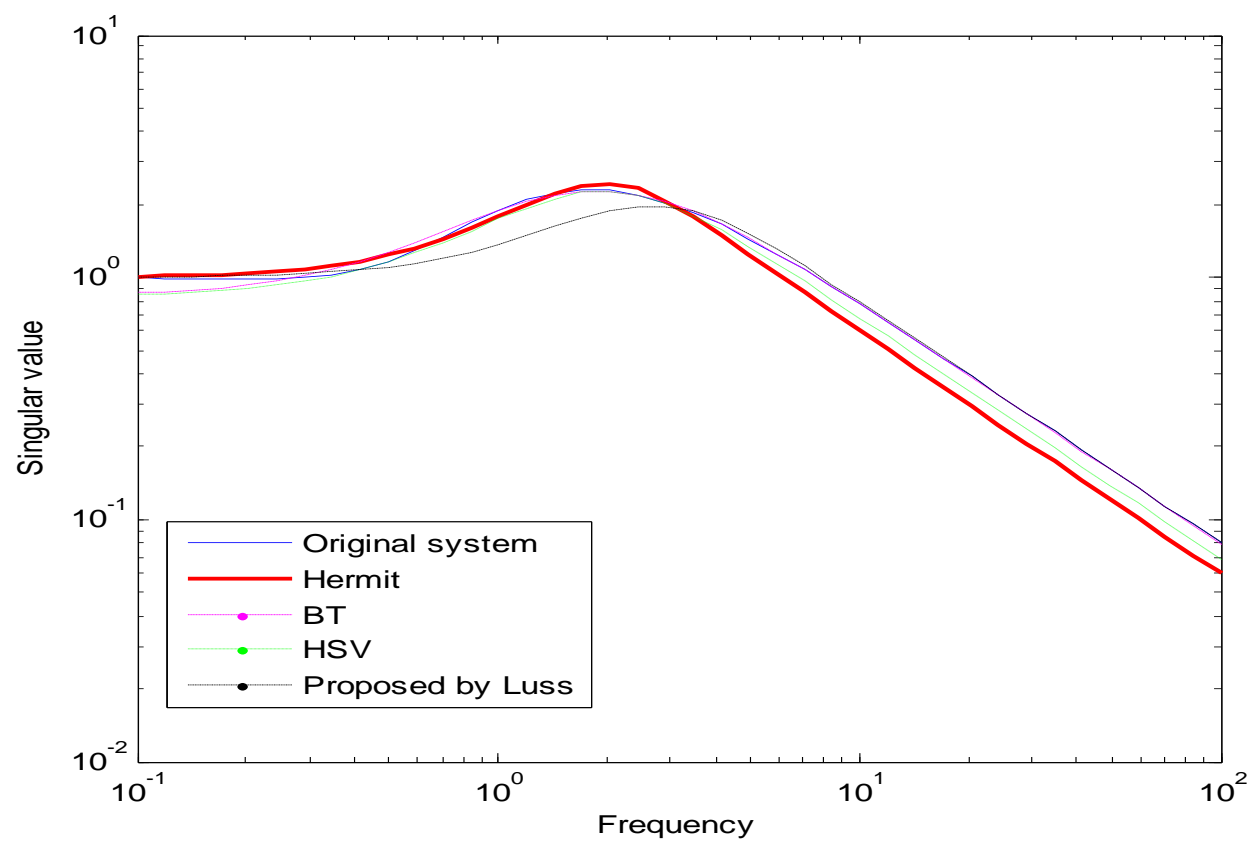

Figure 7: The frequency response of full order and reduced order model by the proposed method and other methods for test system 2.

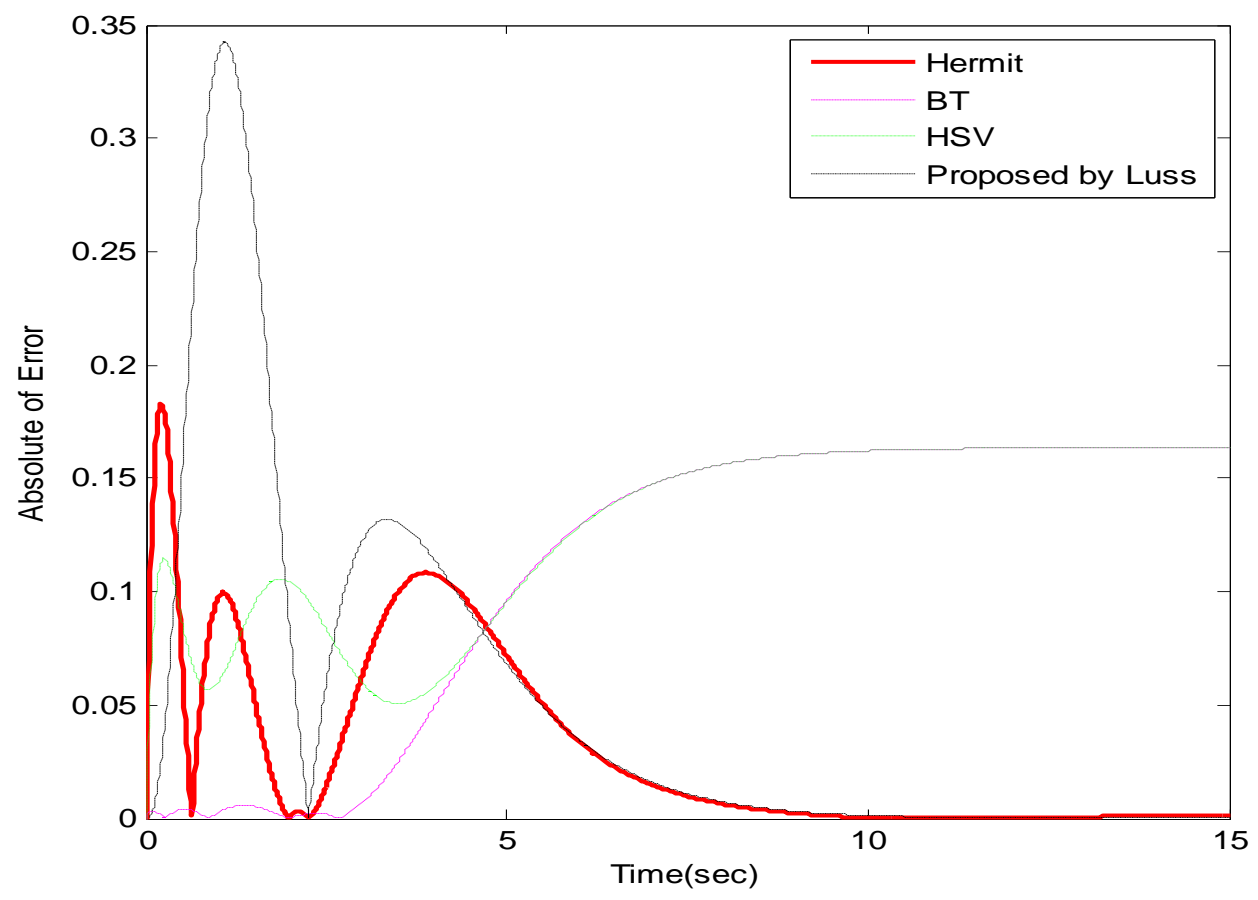

Figure 8: The plot of $e=\left|y-y_{r}\right|$ for the full order and reduced systems by the proposed method and other methods for test system 2. 
Furthermore, the specifications of the proposed method such as maximum overshoot, steady state value, ISE and $H_{\infty}$ norm of the error between the step responses of full order and reduced order models $\left(e=\left|y-y_{r}\right|\right)$ are given in Table 2 .

Table 2. Comparison of methods for test system 2.

\begin{tabular}{|c|c|c|c|c|}
\hline Model & Steady state value & Overshoot $(\%)$ & ISE & $\begin{array}{c}\text { Infinity norm of } \\
\text { error }\end{array}$ \\
\hline Original system & 1 & 86.5 & - & - \\
\hline Hermite - GA & 0.999 & 90.2 & 0.0382 & 0.1824 \\
\hline Proposed by Luss & 1 & 66.1 & 0.1404 & 0.3425 \\
\hline BT & 0.836 & 123 & 0.3802 & 0.1635 \\
\hline HSV & 0.836 & 115 & 0.4043 & 0.1635 \\
\hline
\end{tabular}

Once again, the results obtained confirm that a satisfactory approximation has been achieved. It is clearly seen that the specifications of reduced order model that is achieved by the proposed method are close to the specifications of original system and better than other methods.

Test system 3: The third system to be reduced is a system given in [31] by Mukherjee, where a procedure is presented to obtain reduced system. The system is as follows:

$$
G(s)=\frac{18 s^{7}+514 s^{6}+5982 s^{5}+3638 s^{4}+122664 s^{3}+222088 s^{2}+185760 s+40320}{s^{8}+36 s^{7}+546 s^{6}+4536 s^{5}+22449 s^{4}+67284 s^{3}+118124 s^{2}+109584 s+40320}
$$

Based on the explanations given for test system 1, the obtained reduced system by the proposed method is as follows:

$$
G_{\text {Hermite }}=\frac{16.68 s+5.43}{s^{2}+6.67 s+5.43}
$$

The comparison of the proposed method with the method suggested by Mukherjee in [31], HSV and BT methods are shown in Figs. 9-11 and Table 3, which illustrate that the achieved results from the proposed method is very similar to original system comparing to other methods.

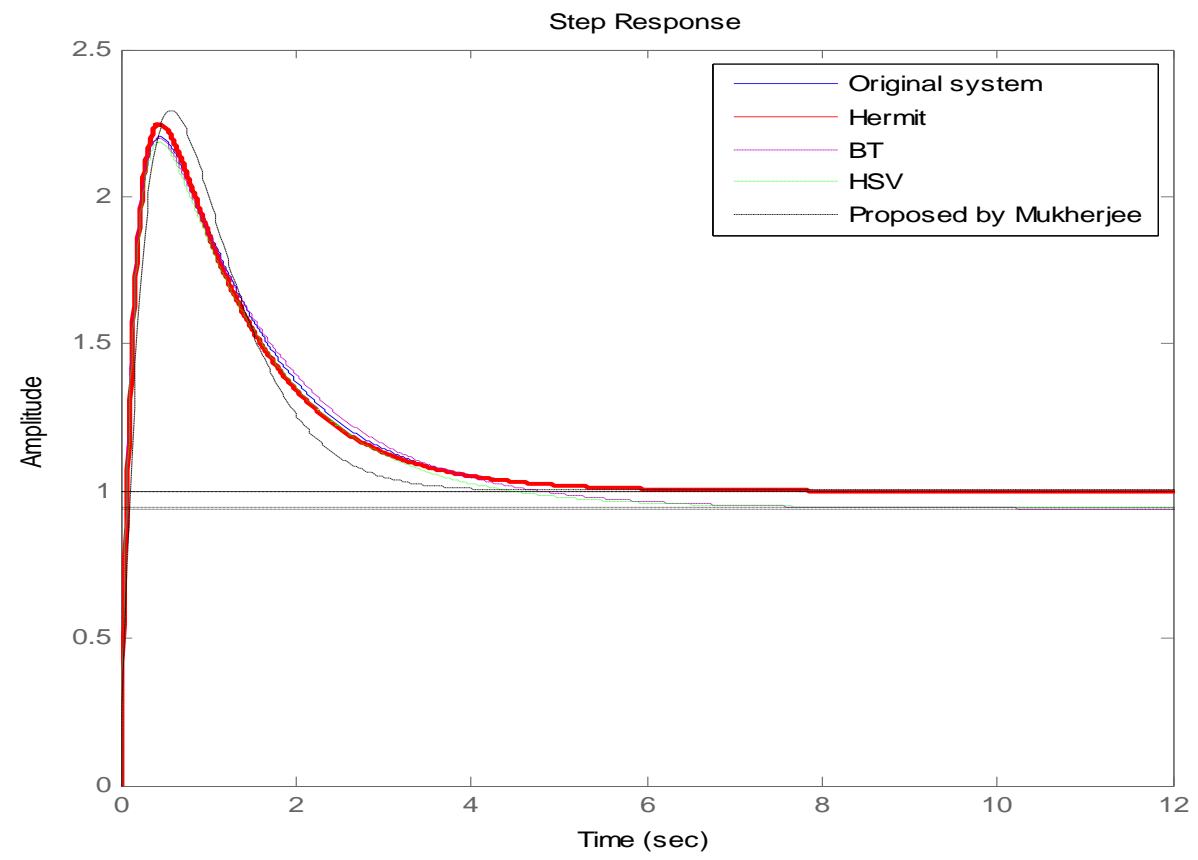

Figure 9: The step response of full order and reduced order model by the proposed method and other methods for test system 3. 


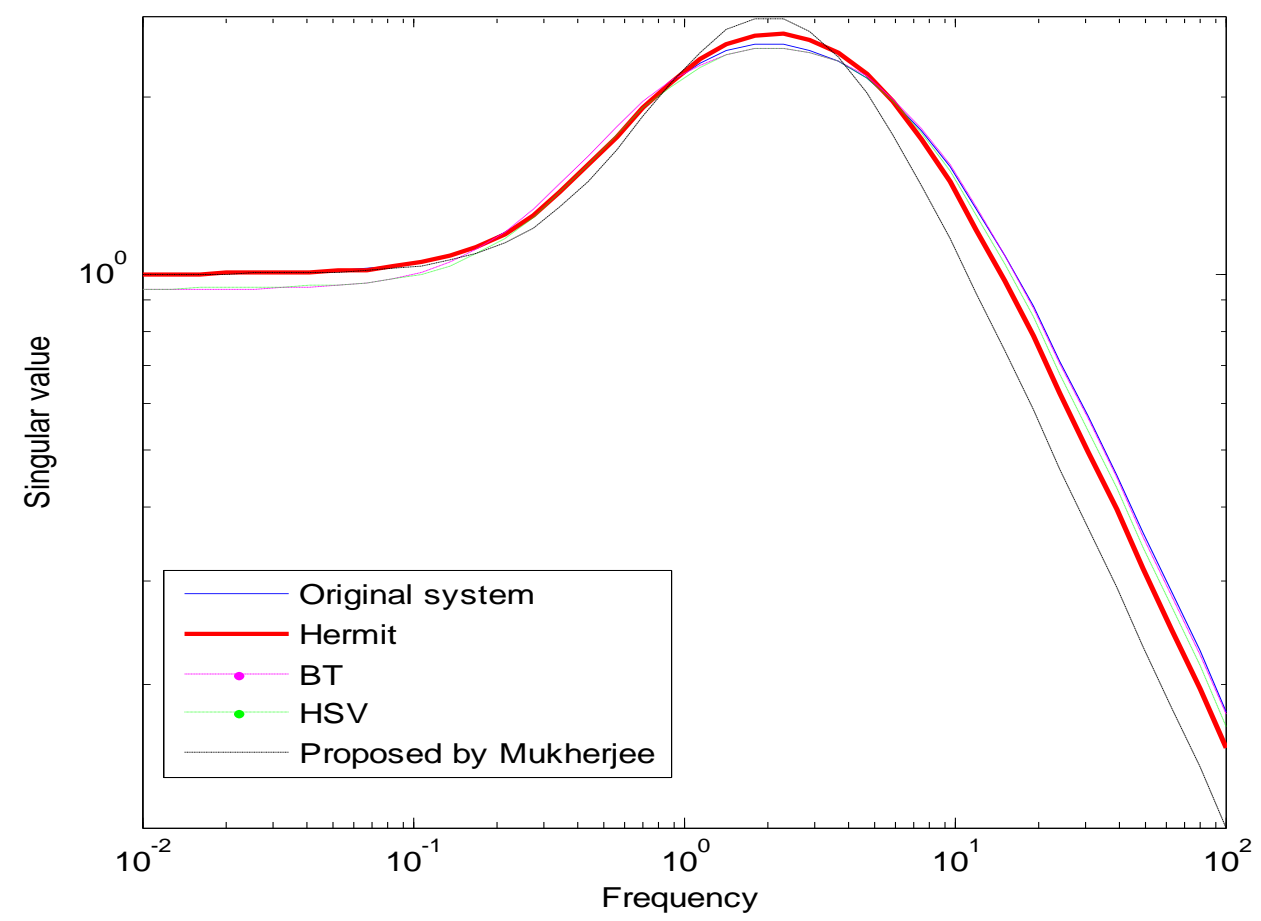

Figure 10: The frequency response of full order and reduced order model by the proposed method and other methods for test system 3.

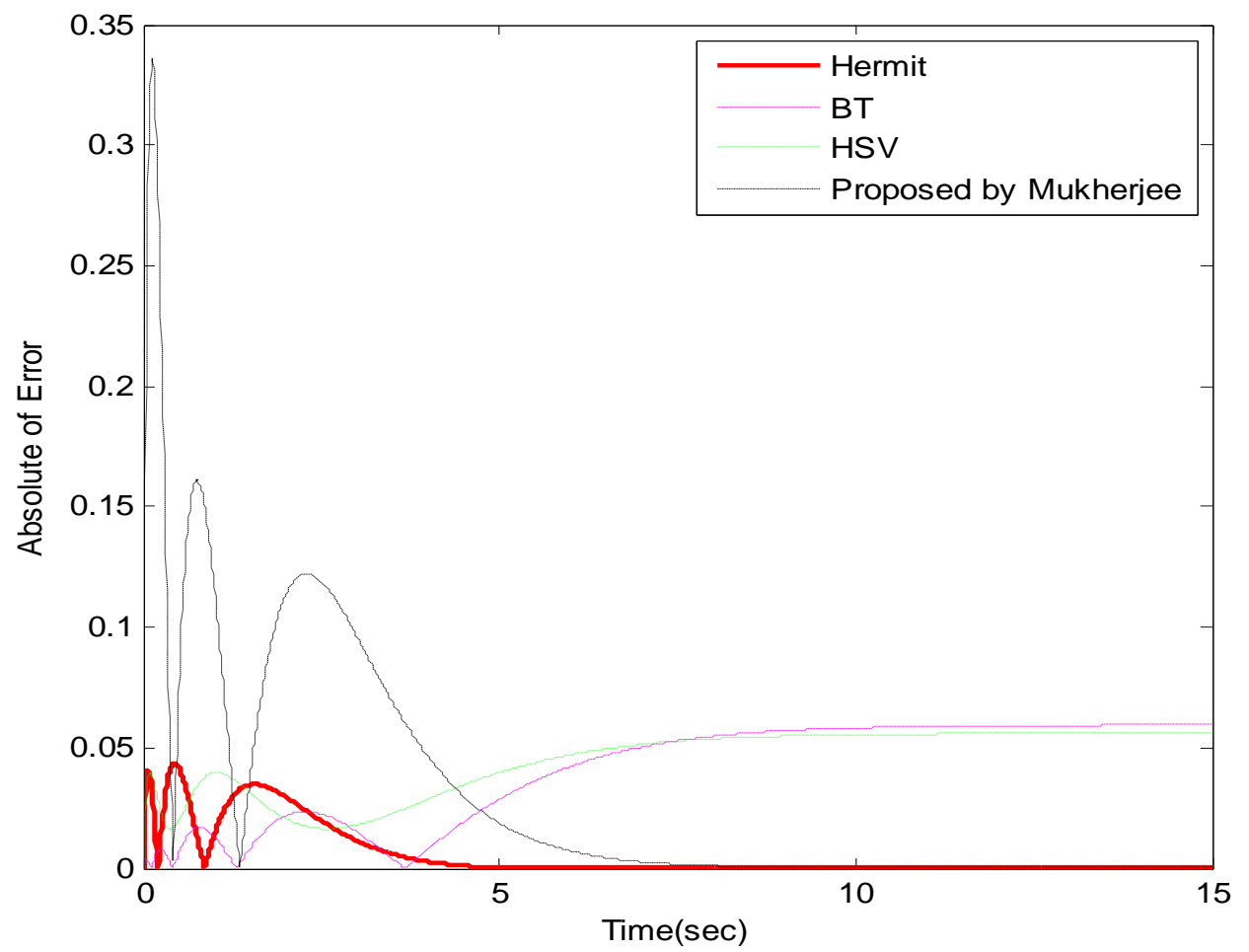

Figure 11: The plot of $e=\left|y-y_{r}\right|$ for the full order and reduced systems by the proposed method and other methods for test system 3 . 
Table 3. Comparison of methods for test system 3.

\begin{tabular}{|c|c|c|c|c|}
\hline Model & Steady state value & Overshoot $(\%)$ & ISE & $\begin{array}{c}\text { Infinity norm of } \\
\text { error }\end{array}$ \\
\hline Original system & 1 & 120 & - & - \\
\hline Hermite - GA & 1 & 125 & 0.0022 & 0.0437 \\
\hline $\begin{array}{c}\text { Proposed by } \\
\text { Mukherjee }\end{array}$ & 1 & 129 & 0.0569 & 0.3361 \\
\hline BT & 0.94 & 134 & 0.0314 & 0.0595 \\
\hline HSV & 0.944 & 132 & 0.0326 & 0.0559 \\
\hline
\end{tabular}

\section{Conclusion}

In this paper, an approach based on orthogonal polynomials using Hermite polynomials and GA is investigated for order reduction. Routh array is applied to determine the stability conditions. To present the accuracy and efficiency of the method, three systems are reduced by the proposed method. The proposed method is compared with some order reduction techniques where the results obtained show that the proposed approach has high accuracy whose results in an adequate low-order model that retains the characteristics of full order model.

\section{References}

[1] E.J. Davison, A Method for Simplifying Linear Dynamic Systems, IEEE Transactions on Automatic Control. 11 (1966) 93 - 101.

[2] M.R. Chidambara, Further Comments by M.R. Chidambara, IEEE Transactions on Automatic Control. 1 (1967) $799-800$.

[3] E.J. Davison, Further Reply by E.J. Davison, IEEE Transactions on Automatic Control. 12 (1967) pp. 800.

[4] M.R. Chidambara, Two simple techniques for the simplification of large dynamic systems, Proc. Joint Automatic Control Conf. (1969) 669-674.

[5] Z. Elrazaz, N.K. Sinha, On the Selection of Dominant Poles of a System to be Retained in a LowOrder Model, IEEE Transactions on Automatic Control. 24 (1979) 792 - 793.

[6] M. Hutton, B. Friedland, Routh Approximations for Reducing Order of Linear, Time-Invariant Systems, IEEE Transactions on Automatic Control. 20 (3) (1975) 329 - 337.

[7] R.K. Appiah, Linear Model Reduction Using Hurwitz Polynomial Approximation, International Journal of Control. 28 (1978) 477 - 488.

[8] R.K. Appiah, Pade Methods of Hurwitz Polynomial with Application to Linear System Reduction, International Journal of Control. 29 (1979) 39 - 48.

[9] T.C. Chen, C.Y. Chang, K.W. Han, Reduction of Transfer Functions by the Stability Equation Method, Journal of the Franklin Institute. 308 (1979) 389 - 404.

[10] T.C. Chen, C.Y. Chang, K.W. Han, Model Reduction Using the Stability Equation Method and the Continued Fraction Method, International Journal of Control. 21 (1980) 81 - 94.

[11] L.G. Gibilaro, F.P. Lees, The Reduction of Complex Transfer Function Models to Simple Models Using the Method of Moments, Chemical Engineering Science. 24 (1969) 85 - 93.

[12] F.P. Lees, The Derivation of Simple Transfer Function Models of Oscillating and Inverting Process from the Basic Transformed Equation Using the Method of Moments, Chemical Engineering Science. 26 (1971) 1179 - 1186.

[13] Y.P. Shih, C.S. Shieh, Model Reduction of Continuous and Discrete Multivariable Systems by Moments Matching, Computer \& System. Engineering. 2 (1978) 127 - 132. 
[14] V. Zakian, Simplification of Linear Time-Variant System by Moment Approximation, International Journal of Control. 18 (1973) 455 - 460.

[15] C.F. Chen, L.S. Shieh, A Novel Approach to Linear Model Simplification, International Journal of Control. 8 (1968) $561-570$.

[16] C.F. Chen, Model Reduction of Multivariable Control Systems by Means Matrix Continued Fractions, International Journal of Control. 20 (1974) 225 - 238.

[17] D.J. Wright, The Continued Fraction Representation of Transfer Functions and Model Simplification, International Journal of Control. 18 (1973) 449 - 454.

[18] Y. Shamash, Stable Reduced-Order Models Using Pade Type Approximation, IEEE Transactions on Automatic Control. 19 (1974) 615 - 616.

[19] D.A. Wilson, Optimal Solution of Model Reduction Problem, Proc. IEEE. 117 (6) (1970) 1161 1165.

[20] D.A. Wilson, Model Reduction for Multivariable Systems, International Journal of Control. 20 (1974) $57-64$.

[21] G. Obinata, H. Inooka, A Method of Modeling Linear Time-Invariant Systems by Linear Systems of Low Order, IEEE Transactions on Automatic Control. 21 (1976) 602 - 603.

[22] G. Obinata, H. Inooka, Authors Reply to Comments on Model Reduction by Minimizing the Equation Error, IEEE Transactions on Automatic Control. 28 (1983) 124 - 125.

[23] E. Eitelberg, Model Reduction by Minimizing the Weighted Equation Error, International Journal of Control. 34 (6) (1981) 1113 - 1123.

[24] R.A. El-Attar, M. Vidyasagar, Order Reduction by $\mathrm{L}_{1}$ and $\mathrm{L}_{\infty}$ Norm Minimization, IEEE Transactions on Automatic Control. 23 (4) (1978) 731 - 734.

[25] B.C. Moore, Principal Component Analysis in Linear Systems: Controllability, Observability and Model Reduction, IEEE Transactions on Automatic Control. 26 (1981) 17-32.

[26] L. Pernebo, L.M. Silverman, Model Reduction via Balanced State Space Representation, IEEE Transactions on Automatic Control. 27 (2) (1982) 382 - 387.

[27] D. Kavranoglu, M. Bettayeb, Characterization of the Solution to the Optimal Hळ Model Reduction Problem, Systems and Control Letters. 20 (1993) 99 - 107.

[28] L. Zhang J. Lam, On $\mathrm{H}_{2}$ Model Reduction of Bilinear System, Automatica. 38 (2002) 205 - 216.

[29] W. Krajewski, A. Lepschy, G.A. Mian, U. Viaro, Optimality Conditions in Multivariable L2 Model Reduction, Journal of the Franklin Institute. 330 (3) (1993) 431 - 439.

[30] D. Kavranoglu, M. Bettayeb, Characterization and Computation of the Solution to the Optimal $\mathrm{L}_{\infty}$ Approximation Problem, IEEE Transactions on Automatic Control. 39 (1994) 1899 - 1904.

[31] G. Parmar, S. Mukherjee, R. Prasad, Reduced Order Modeling of Linear Dynamic Systems using Particle Swarm Optimized Eigen Spectrum Analysis, International Journal of Computer and Mathematic Science. 1 (1) (2007) 45 - 52.

[32] G. Parmer, R. Prasad, S. Mukherjee, Order Reduction of Linear Dynamic Systems using Stability Equation Method and GA, World Academy of Science, Engineering and Technology. 26 (2007) $72-78$.

[33] Hasan Nasiri Soloklo, M. Maghfoori Farsangi, Multi-Objective Weighted Sum Approach Model Reduction by Routh-Pade Approximation Using Harmony Search, Turkish journal of Electrical Engineering and Computer Science. 21 (2013) 2283-2293.

[34] S. Panda, J.S. Yadav, N.P. Padidar, C. Ardil, Evolutionary Techniques for Model Order Reduction of Large Scale Linear Systems, International Journal of Applied Science Engineering Technology. 5 (2009) 22 - 28.

[35] G. Parmar, M.K. Pandey, V. Kumar, System Order Reduction Using GA for Unit Impulse Input and a Comparative Study Using ISE and IRE, International Conf. Advance in computing, communications and control. (2009), Mumbai, India.

[36] R. Salim, M. Bettayeb, $\mathrm{H}_{2}$ and $\mathrm{H}_{\infty}$ Optimal Reduction Using Genetic Algorithm, Journal of the Franklin Institute. 348 (2011) 1177 - 1191. 
[37] H. Nasiri Soloklo, M. Maghfoori Farsangi, Chebyshev Rational Functions Approximation for Model Order Reduction Using Harmony Search, Scientia Iranica. 30 (3) (2013) 771-777.

[38] E. Kreyszig, Advanced Engineering Mathematics, John Wiley, New York, 1999.

[39] M. Vatani, M. Asghari, Gh. Vakili-Nezhaad, Application of Genetic Algorithm to Parameter Estimation in Liquid-Liquid Phase Equilibrium Modeling, The Journal of Mathematics and Computer Science. 5 (1) (2012) 60-66.

[40] V. Singh, Obtaining Routh-Pade Approximants using the Luus-Jaakola Algorithm, IEE Proceeding, Control Theory and Applications. 152 (2) (2005) 129 - 132.

[41] S. Mukherjee, Satakhshi, R.C. Mittal, Model Order Reduction Using Response- Matching Technique, Journal of the Franklin Institute. 342 (2005) 503 - 519.

[42] S. Skogestad, I. Postethwaite, Multivariable Feedback Control, Analysis and Design, John Wiley, 1996. 